Revue des patrimoines

$26 \mid 2015$

La reconversion des sites et des bâtiments industriels

\title{
La reconversion des sites et des bâtiments industriels
}

Éditorial

Paul Smith

\section{OpenEdition}

\section{Journals}

Édition électronique

URL : http://journals.openedition.org/insitu/11802

DOI : 10.4000/insitu. 11802

ISSN : 1630-7305

Éditeur

Ministère de la Culture

Référence électronique

Paul Smith, «La reconversion des sites et des bâtiments industriels », In Situ [En ligne], 26 | 2015, mis en ligne le 07 juillet 2015, consulté le 22 septembre 2020. URL : http://journals.openedition.org/insitu/ 11802 ; DOI : https://doi.org/10.4000/insitu. 11802

Ce document a été généré automatiquement le 22 septembre 2020.

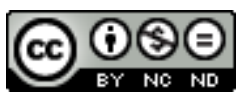

In Situ Revues des patrimoines est mis à disposition selon les termes de la licence Creative Commons Attribution - Pas d'Utilisation Commerciale - Pas de Modification 4.0 International. 


\section{La reconversion des sites et des bâtiments industriels}

Éditorial

Paul Smith

1 Sous des noms variés - conversion, reconversion, réhabilitation, réutilisation, recyclage, transformation, mutation, transcription... -, l'adaptation de sites et de bâtiments délaissés par leurs fonctions premières de production industrielle est aujourd'hui monnaie courante dans tous les pays d'ancienne industrialisation. Née dans les années 1970 avec l'émergence d'une nouvelle sensibilité à l'égard des vestiges physiques de l'industrialisation, la reconversion s'est imposée, faute de pouvoir créer partout des musées de l'industrie, comme une démarche susceptible de préserver quelque chose des valeurs de témoignage de ce patrimoine. Elle s'est parée depuis lors de nouvelles vertus sous les enseignes du développement durable et de la requalification urbaine. 
Figure 1

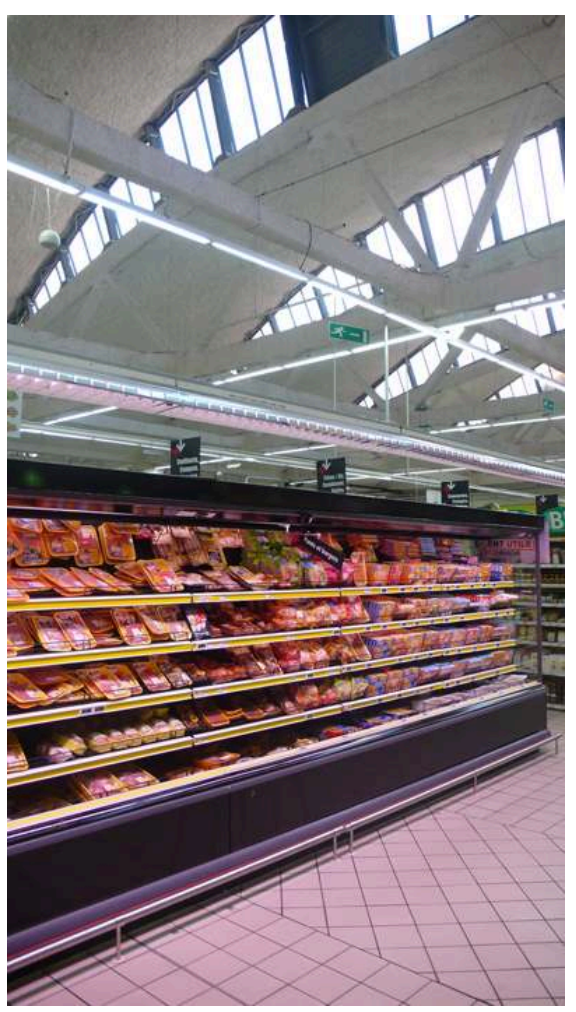

Aulnay-sous-Bois (Seine-Saint-Denis), ancienne usine de chauffage central Idéal Standard, atelier des grosses chaudières converti en supermarché au début des années 1980.

Phot. Smith, Paul. (c) Paul Smith.

2 Pour les professionnels et les amis du patrimoine industriel, cette question de la reconversion semble constituer l'un des quatre grands enjeux auxquels ce patrimoine se trouve confronté en ce premier quart $\mathrm{du} \mathrm{xxI}^{\mathrm{e}}$ siècle. Le premier de ces enjeux demeure la poursuite, sur les plans national et régional, de l'effort méthodique d'accumulation de connaissances sur ce patrimoine, l'approfondissement de ces connaissances et leur partage avec un public aussi large que possible, celui en particulier des décideurs au sein des collectivités territoriales. L'enseignement autour du patrimoine de l'industrie et la formation à ses métiers - et à ses plaisirs - de nouvelles générations de chercheurs et de spécialistes est un aspect essentiel de cet impératif. 
Figure 2

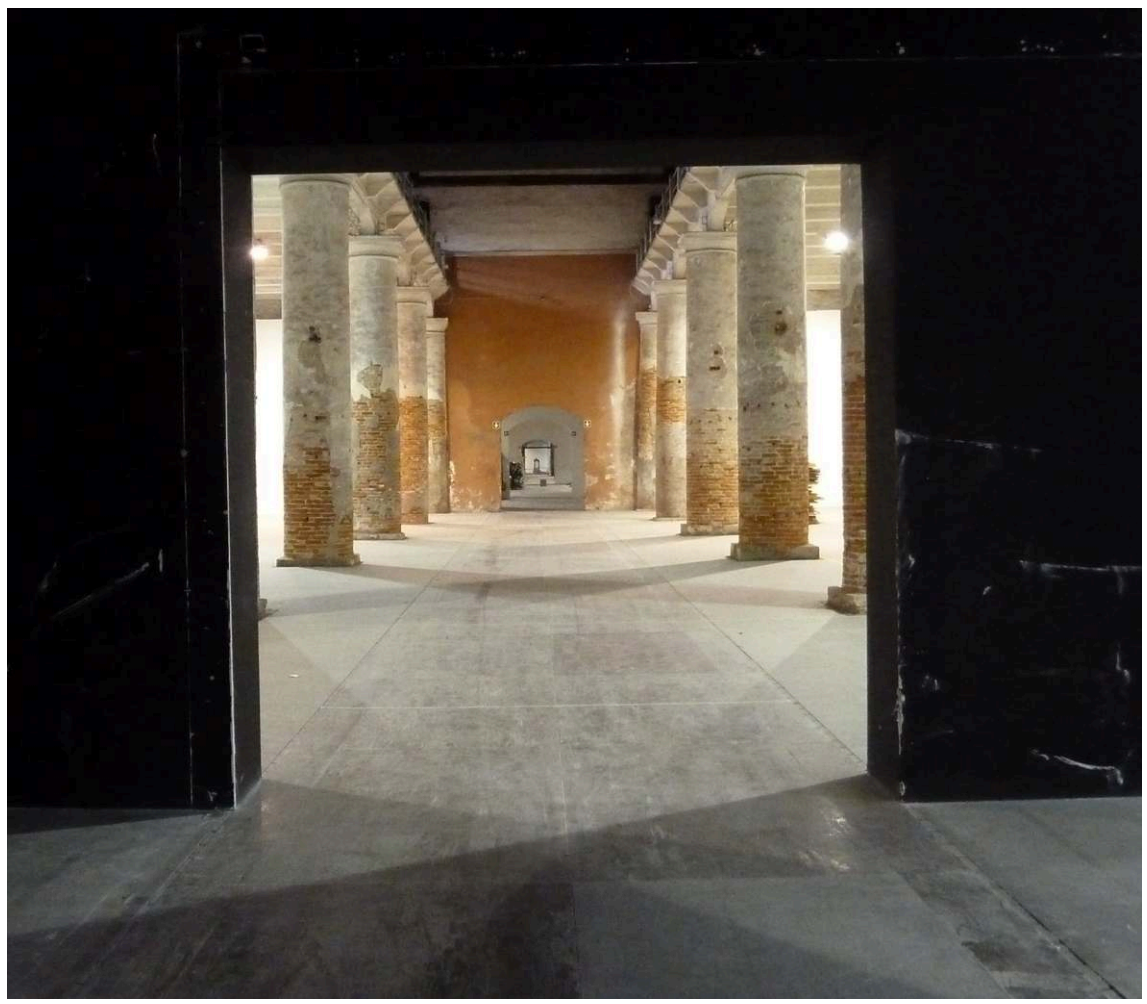

Venise, corderie de l'arsenal construite de 1576 à 1585, suivant les dessins d'Antonio da Ponte. Les 6400 mètres carrés de surface sont utilisés de nos jours par la Biennale d'Art.

PHOT. SMITH, PAUL. (C) PAUL SMITH.

3 Le deuxième enjeu concerne l'étude plus particulière et la sauvegarde raisonnée du patrimoine des industries $\mathrm{du} \mathrm{xx}^{\mathrm{e}}$ siècle, un patrimoine sans doute moins aisément apprécié que celui des siècles antérieurs et, par la nature même de ses formes construites - on pense ici aux installations plus proches en réalité de la machine que du bâtiment, comme les silos, les hauts fourneaux, les gazomètres, les tours à plomb ou à trempe, les lavoirs de charbon, les raffineries -, d'une conservation et d'une réinsertion économique moins évidentes.

4 Le troisième enjeu, proche du précédent, est celui de la sauvegarde des machines de production industrielle, bien plus difficile que celle des bâtiments qui les abritaient. En effet, au moment où l'on engage l'étude de ces bâtiments et que démarre un processus de patrimonialisation à leur égard, les machines, sauf cas exceptionnel, sont parties depuis longtemps à la ferraille. La très faible représentation de ce patrimoine technique de l'industrie dans la base Palissy des objets mobiliers souligne cet état de fait. 


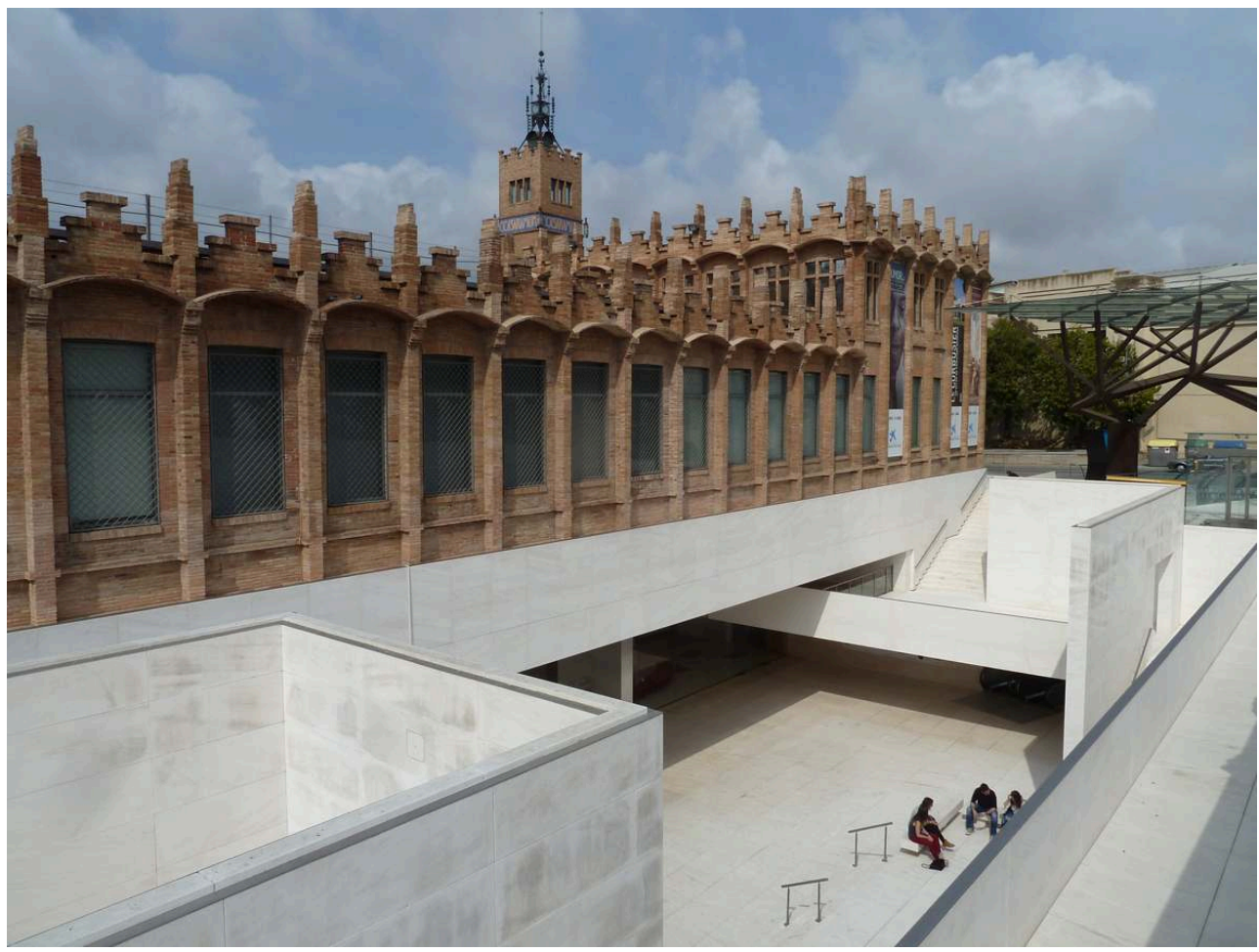

Barcelone, ancienne usine textile Casaramona, dessinée par l'architecte Josep Puig i Cadafalch et construite de 1909 à 1912, transformée depuis 2002 en centre culturel CaixaForum.

Phot. Smith, Paul. (c) Paul Smith.

5 Le quatrième enjeu, enfin, est celui de la reconversion du patrimoine industriel, l'invention pour les anciens sites et bâtiments de production de nouveaux usages qui ne gommeraient pas leur histoire, n'occulteraient pas leurs valeurs de témoignage. Plusieurs initiatives - notamment le colloque international «Patrimoine industriel, nouvelles politiques urbaines et sens de la reconversion » organisé à Belfort en 2011', un autre colloque international sur la reconversion des manufactures des tabacs en Europe, organisé à Nantes en 2013, et, enfin, une exposition récente à la Cité de l'Architecture et de Patrimoine, "Un bâtiment, combien de vies? $»^{2}$ - suggèrent l'actualité de cette thématique. Bien au-delà des milieux des amis du patrimoine industriel, elle préoccupe des architectes, des urbanistes, des aménageurs et des élus, sans oublier le monde des créatifs, colonisateurs de ces «nouveaux territoires de l'art ».

6 Pour tous ces acteurs, la reconversion des bâtiments industriels renvoie d'une manière ou d'une autre à la question des "bonnes pratiques». Quelles opérations de reconversion sont à mettre en exergue pour leur exemplarité ? Ici, bien évidemment, les appréciations divergent. Pour l'ami du patrimoine industriel, la réussite d'une opération relèverait de considérations comme le choix d'un programme approprié à la morphologie des bâtiments reconvertis, le respect des matériaux de ces bâtiments ou encore l'intégration dans l'opération d'un espace consacré à l'interprétation de l'histoire du site. Pour un architecte, la réussite peut être une question d'équilibre et d'harmonie entre le bâti neuf et le déjà-là ; pour un aménageur, l'impact positif de l'opération sur son environnement urbain; pour un promoteur, l'économie globale de l'opération, comprenant le cachet supplémentaire qu'apporte un patrimoine préservé ; 
pour un élu, les retombées médiatiques et la contribution à l'image et à l'attractivité de sa ville ; pour un usager, un environnement agréable à voir et à vivre...

Figure 4

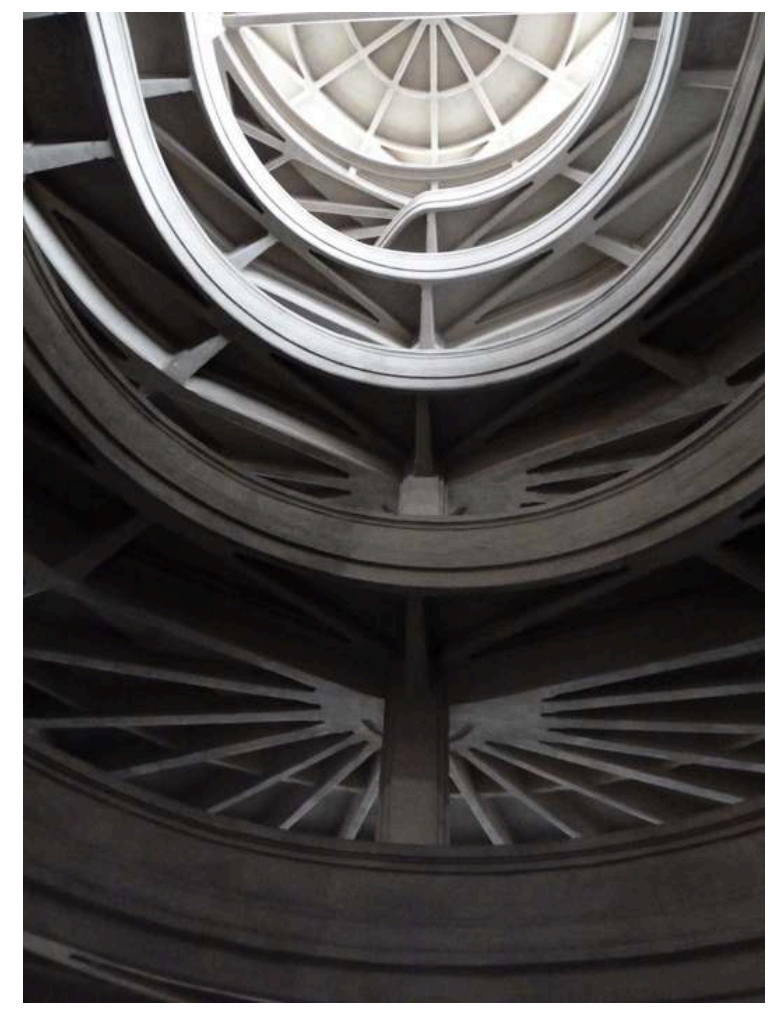

Turin, ancienne usine Fiat de Lingotto, dessinée en 1915 par l'ingénieur Giacomo Mattè Trucco, reconvertie à partir de 1985 par l'architecte Renzo Piano pour de nombreux usages culturels et commerciaux; vue de la rampe hélicoïdale donnant accès à la célèbre piste d'essais sur le toit de l'édifice.

Phot. Smith, Paul. (c) Paul Smith.

$7 \quad$ C'est de cette diversité de points de vue que découle l'idée de ce numéro thématique de la revue In Situ. Sans avoir la prétention d'énoncer des bonnes pratiques en la matière, le projet était de demander à différents auteurs de présenter une opération de reconversion du patrimoine industriel qui leur semblait réussie, en explicitant les termes de cette réussite. Un appel à communications diffusé en 2011 espérait ainsi aboutir à une compilation d'articles «coup de cœur», genre "ma reconversion préférée ».

8 Sans être aussi nombreux qu'on l'avait souhaité, les articles rassemblés ici présentent un véritable intérêt de par leurs qualités intrinsèques et par leur mise en résonance. On peut noter en particulier la diversité géographique des sites et des bâtiments examinés - Bordeaux, Rome, Budapest et New York - ainsi que la diversité des types de patrimoine abordés : des chais de stockage, une centrale électrique, une grande usine de construction électrique et un viaduc ferroviaire urbain. Les auteurs demandent toutefois qu'il soit bien précisé ici que leurs textes ont maintenant trois ans, d'où peutêtre quelques évolutions récentes qui n'ont pas pu être prises en compte.

9 Enfin, ce numéro thématique de la revue (qui pourra s'enrichir dans les mois à venir de nouvelles contributions) s'ouvre avec un survol général de la question de la reconversion du patrimoine industriel rédigé par Emmanuelle Real et constituant à 
l'origine l'introduction de sa publication sur des exemples de reconversions dans la région où elle travaille ${ }^{3}$. Grâce à la reproduction du texte dans cette revue électronique, il trouvera, espérons-le, les lecteurs qu'il mérite, au-delà de la Haute-Normandie.

\section{Figure 5}

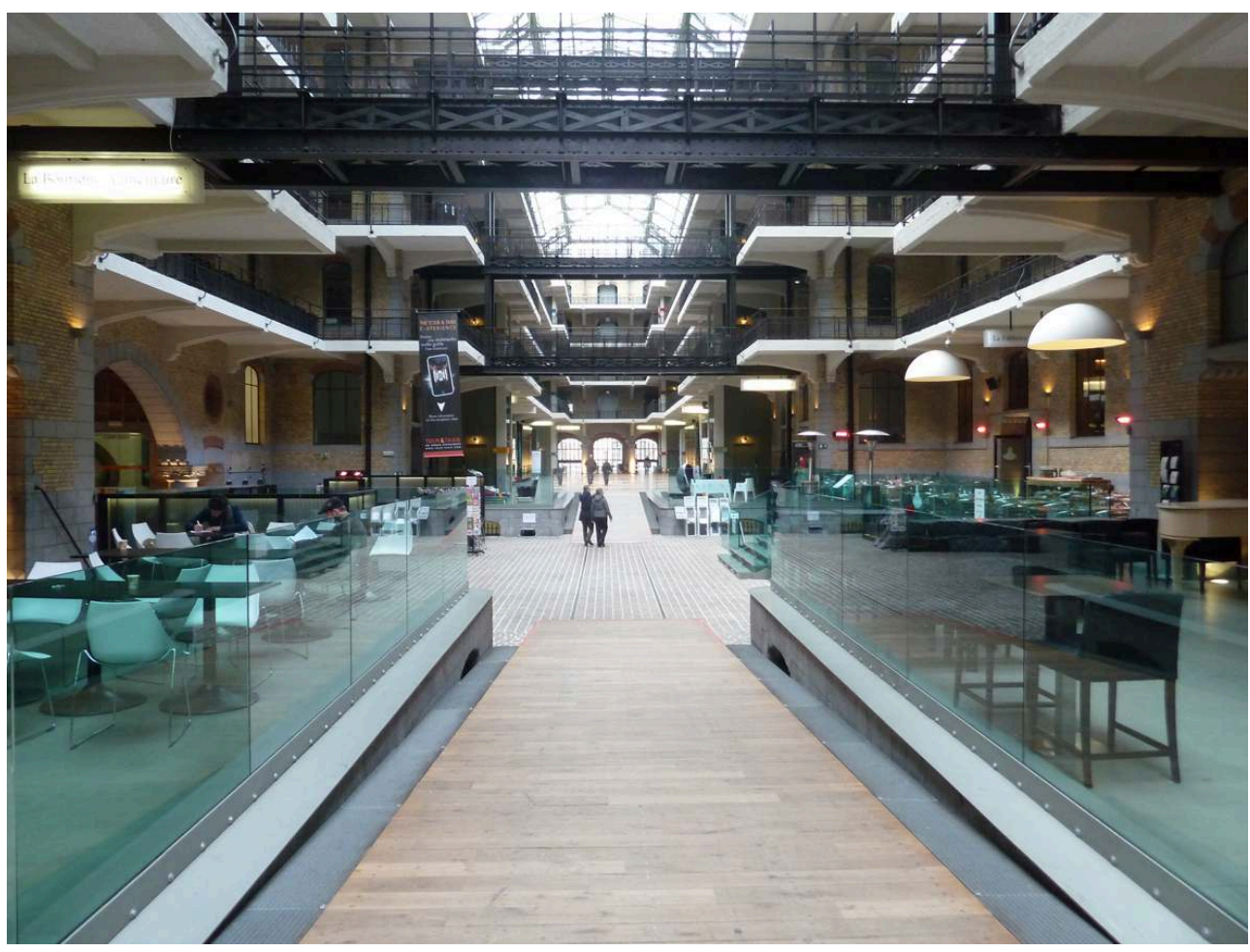

Bruxelles, l'ancien entrepôt royal Tour \& Taxis, construit de 1904 à 1906 selon les plans de l'architecte Van Humbeek, accueille aujourd'hui des entreprises et des commerces.

Phot. Smith, Paul. (c) Paul Smith.

Nous remercions très vivement Catherine Gros pour son travail de relecture des textes.

\section{NOTES}

1. - Les actes de ce colloque, co-organisé par le laboratoire Récits de l'université de technologie de Belfort-Montbéliard, la direction générale des Patrimoines au ministère de la Culture et de la Communication et le CILAC (Comité d'information et de liaison pour l'archéologie, l'étude et la mise en valeur du patrimoine industriel), sont publiés dans L'Archéologie industrielle en France, $\mathrm{n}^{\circ}$ 60, juin 2012.

2. - RAMBERT, Francis (dir.). Un bâtiment, combien de vies ? La transformation comme acte de création. Paris : Cité de l'architecture et du patrimoine, 2014.

3. - REAL, Emmanuelle. Reconversions, l'architecture industrielle réinventée, Haute-Normandie. Rouen : Conseil régional de Haute-Normandie, 2013. 


\section{AUTEUR}

\section{PAUL SMITH}

Département du pilotage de la recherche et de la politique scientifique, Direction générale des Patrimoines, Ministère de la Culture et de la Communication paul.smith@culture.gouv.fr 